\title{
El estatus y la función de lo religioso en la Academia como debate social. \\ Visión desde la Universidad*1
}

\author{
Pierre Gisel \\ PROFESOR DE LA FACULTAD DE TEOLOGÍA \\ Y CIENCIAS DE LA RELIGIÓN DE LA UNIVERSIDAD DE LAUSANA, SUIZA.
}

1. La Universidad como institución política y civil descentrada de las instituciones religiosas

El título de mi primer punto refleja una visión de fondo que afecta a la sociedad y lo que en ella se despliega, partiendo de la base de que nuestras sociedades modernas están atravesadas por una diferenciación de esferas culturales: el saber, lo religioso, lo estético, lo moral, lo penal, teniendo en el trasfondo tanto lo político como lo civil como esferas que se distinguen entre sí. Se trata de esferas que poseen sus propias regulaciones y que dependen de racionalidades diferentes. Abordar este punto implica esclarecer aspectos, tales como lo que está en juego en cada una de esas esferas en términos del «interés» humano y social. La perspectiva de fondo que propondré se vincula también a la pluralidad que, en nuestras sociedades, atraviesa cada una de las esferas mencionadas, pluralidad de las referencias, los objetivos, los tipos de posicionamiento.

Lo político ejerce en esta materia una función de árbitro. Ciertamente, se ocupa también del «bien común», pero entendiendo que el «bien común»-una noción antigua por la cual conserva su preocupa-

* Nota del editor. Que esta conferencia, pronunciada en francés, fuera publicada en traducción castellana, fue condición puesta por su autor. La traductora fue Patricia Concha.

1 Texto de una ponencia presentada con motivo de la ceremonia de inauguración del Centro de Estudios de la Religión de la Pontificia Universidad Católica de Chile el 5 noviembre 2015 (el título y subtítulos fueron propuestos por los organizadores, especialmente la Profesora Sandra Arenas). 
ción lo mejor de la tradición católica- difiere de un programa social y humano ideal, que debe promoverse, o incluso imponerse, para el bien o el bienestar de todos, en cuyo caso resulta una tentación recurrente presente en la esencia misma de los tiempos modernos. Tradicionalmente, el «bien común» se entiende como lo que permite a cada uno y a cada organización su "propia perfección». Remite entonces a «fines» que no son homogéneos en lo político -ni incluso en lo social como tal-, sino que lo eluden y lo sobrepasan, fines todos irreductiblemente particulares.

Árbitro en primer lugar, y, por lo tanto, neutral, lo político, específicamente el Estado, será en principio laico y su responsabilidad en términos de bien "positivo" será la de favorecer la "respiración" de la sociedad civil respetando la expresión de sus diferencias. Ello supone un espacio pacificado -tradicionalmente, una de las tareas del Estado es velar por ello-, aunque esto no debiera llevar a reprimir el debate, aun cuando sea áspero y agudo, respecto de asuntos de fondo que son objeto de disenssus. En efecto, también es tarea del Estado velar por que las diferentes maneras de dar forma al despliegue de lo humano -y, por lo tanto, a las diferencias en materia de tradiciones, referencias y objetivos- se vuelvan fructíferas en la medida en que su exposición ante otros, se confronta, se desarrolla y comparte en términos humanos y de convivencia. Por otra parte, esto redundará en beneficio de cada uno y de todos, aunque sea de manera indirecta, lejos de toda reducción de las diferencias o de su equiparación. Plantear las cosas en estos términos ciertamente implica situarse fuera de una matriz teológico-política -teocrática en las versiones más duras-, pero a la vez fuera de volcarse a un laicismo puro y simple, en menor o mayor grado sustitutivo, aun cuando este haya sido concebido como una emancipación valiosa ${ }^{2}$.

El laicismo es para mí un concepto pertinente y necesario para lo político. Pero evitaremos ampliarlo a la sociedad como tal -a lo civil-, atravesada, entre muchos otros factores, por lo religioso y lo religioso diverso. Agrego que la distinción entre lo político y lo civil, así como la sanción otorgada y por otorgar a la pluralidad en el plano civil, van de

2 En esta materia, y en relación con lo que atraviesa nuestras sociedades contemporáneas, remito a mi artículo "Mutations du théologico-politique. Quels déplacements, quels défis, quelles tâches ? ", Archives de sciences sociales des religions 169, 2015/1, p. 63-83. 
la mano con lo que se inscribe justamente en lo civil: una diversidad de instituciones específicas, «intermediarias» entre la persona y el Estado. Pueden ser de diferente orden y considerarse cada vez y articularse, tanto en lo civil en su conjunto como en las realidades específicamente políticas. A título de ejemplo, consideraremos aquí las Iglesias u otras comunidades religiosas, vinculadas a la tradición o a maneras de hacerse cargo de lo humano, simbolizarlo, situarlo y darle un relato. Pero pensaremos también en la Universidad, que no estará bajo las órdenes del Estado, aun cuando sea el Estado el que la financie (el Estado financia la justicia sin, por ello, convertirse en su dueño ni, en principio, darle instrucciones, fuera del marco formal). El Estado financia la Universidad porque estima que su existencia misma, en su propia diferencia y en una cierta autonomía, es buena para la sociedad en la cual tiene la responsabilidad de favorecer el bienestar. Con ello, se subentiende que la Universidad remite a una instancia distinta de lo político, tal como la justicia remite a una instancia distinta de lo político -e incluso de lo social como tal-, aquella que cristaliza el derecho. Para la Universidad, esa instancia es la del saber -ya sea que guste o no lo que se encuentre asegurado o confirmado o ya sea moral o no-, siempre y cuando el saber o, de hecho, los saberes, dependan de diversos regímenes de razón, especialmente, en los asuntos sociales y humanos, pero todos descentrados y no reductibles a la conciencia y a la voluntad de los actores. Esto es válido tanto para lo religioso como para toda otra cosa.

Ese era, a mi parecer, el primer punto a plantear. Por lo tanto, no parto de una apreciación que considera dos instancias -lo religioso y lo político, o la Iglesia y el Estado- más o menos en confrontación y en competencia, al menos parcial -que presentan conflictos sobre sus límites-, ni tampoco de una apreciación que considera como complementarias dichas instancias. Son, pues, distintas: no pertenecen al mismo espacio ni sancionan datos heterogéneos. Por tanto, van a desplazar, para efectos de pensarlas inscribiéndolas en un juego más complejo y diferenciado, un juego social no homogeneizado ni homogeneizable y que, más aún, no debe ser homogeneizado, ni siquiera de manera ideal, lo que no excluye los respectivos aportes de una instancia a la otra.

Es claro que este marco que descentra de manera patente las realidades y los enfoques de lo religioso implica que el tratamiento de lo religioso en la Universidad no puede reducirse a las formas de lo que se hace en las facultades de Teología, vinculadas a una u otra tradición 
confesional, incluso pese a que se trabaje la realidad de manera crítica y abierta $^{3}$. Más adelante, señalaré por qué y en qué, en este marco global, un apoyo a entidades académicas del tipo de las facultades de Teología me parece, no obstante, posible y legítimo de parte del Estado, en virtud de disposiciones jurídicas diversas, aunque dicho apoyo debiera materializarse sin traspasar el marco indicado y precisando, específicamente en dicho marco, su objetivo y función. Ahora bien, antes de abordar este punto ${ }^{4}$, me referiré brevemente a los diversos aspectos de los desafíos sociales vinculados a lo religioso que debe enfrentar la Universidad, para luego abordar, de manera más directa, la cuestión de saber cuándo lo religioso, como tal, se plantea como el espacio de un debate social y, recíprocamente, cuándo el debate social, como tal, puede plantearse pleno de cuestiones de hecho religiosas o que, al menos, hayan podido ser impulsadas por las religiones.

\section{Lo que debe conocerse}

El primer dato que debemos registrar es que lo religioso es un campo de realidades muy diversas. Comprende desde tradiciones diferentes, pero también aspectos distintos a las tradiciones, hasta creaciones modernas (la Cientología, los Raelianos, entre otros). Lo religioso es también difuso, como lo demuestran movimientos tipo New Age, la moda de los "caminos de vida», las propuestas que encuentran hoy en día gran eco, como las «espiritualidades sin Dios» o "espiritualidades laicas» ${ }^{5}$, que reflejan todas el modo en que se traspasan las fronteras que podrían circunscribir lo que es lo religioso ${ }^{6}$. Este es un aspecto de lo que ocurre en

3 La facultad en la cual me desempeñé durante treinta y seis años como profesor ha dado un vuelco institucional de un estado de facultad de Teología, de tradición protestante, a un estado de facultad de Ciencias de las Religiones y ello ha sido ocasión de debates importantes e instructivos; ver, en esta materia, el pequeño libro que publiqué luego del clímax de estos acontecimientos, Traiter du religieux à l'Université. Une dispute socialement révélatrice, Lausanne, éd. Antipodes, 2011.

4 Ver más adelante la nota 25 y el pasaje de mi texto con el cual se relaciona.

5 Como ejemplo, consultar André Comte-Sponville, L'esprit de l'athéisme. Introduction à une spiritualité sans Dieu, Paris, A. Michel, 2006, o Luc Ferry, La Révolution de l'amour. Pour une spiritualité lä̈que, Paris, Plon, 2010.

6 Ver a este respecto mi artículo Qu'est-ce qu'une religion? Paris, Vrin, 2007, ya desde su primer punto (traducción italiana : Che Cosa è una religione?, Brescia, Editrice Queriniana, 2011). 
la esencia de nuestras sociedades posmodernas, pero la frontera que delimita lo que sería religioso de lo que no lo sería está, de hecho, marcada históricamente y, por ello, es cambiante. La distinción entre el contexto cultural y los recursos religiosos, por ejemplo, no es pertinente en el hinduismo, así como en las religiones denominadas étnicas que exploran los antropólogos, y la relación con el cosmos, sus leyes y equilibrios, que presenta el taoísmo, se desarrolla también fuera de esta distinción. En su historia de larga data, el Occidente refleja igualmente diferencias y discontinuidades que abordan el estatus y la función de lo religioso $-y$, por ende, su relación con otras realidades humanas y sociales-, de la Antigüedad clásica a la Antigüedad tardía, ciertamente -y no se trata solamente, ni en primer lugar, de que el cristianismo se desarrolle o nazca allí-, sino especialmente, en el transcurso de la historia misma del cristianismo, de la Antigüedad tardía a la Edad Media, a los tiempos modernos o a la posmodernidad, también diferente a su vez.

La función -y por ello el estatus- de lo que podemos denominar lo religioso no cambia solamente según el espacio que cubran las diferentes civilizaciones o los sistemas simbólicos; sino que cambia también con el paso del tiempo. Lo refleja, para comenzar, en nuestra historia occidental, la dualidad de los dos verbos asociados a religio: relegere (una actitud de retiro, «sabia» y de relectura ante un cosmos que rebasa lo humano y del cual conviene leer los «signos», una «virtud», que se opone al hubris) y religare (vincularse verticalmente con Dios y horizontalmente con los hombres, entre ellos, la comunidad o la Iglesia). A ello se agrega toda la gama de relaciones existentes entre religión y poderes donde solo mediante una perspectiva corta y determinada por los afectos modernos puede pensarse que son y han sido siempre cómplices y, por lo mismo, una legitimización recíproca en un conjunto en principio unitario. Finalmente, aludiremos a los puntos de convergencia entre «esferas» diferentes, tan verdaderas como lo simbólico y lo ritual, centrales en materias religiosas, que se encuentran y funcionan justamente fuera de la religión, en política, en la vida cotidiana, individual y social, en el deporte y en otros ámbitos.

En materias religiosas, existen diferencias irreductibles: en efecto, la disposición misma del terreno es distinta, no solamente se trata de la manera de ocupar los lugares que se encontrarían en cada uno (una referencia a un dios o a lo divino, afirmación de un fundamento, un sistema de mediaciones, una organización de tipo comunitario, etc., todos ellos 
aspectos que no se presentan justamente en toda circunscripción religiosa). Asimismo, existen discontinuidades en la forma de cambios de dirección, que no constituyen un simple juego que se modifica y retoma cuando es posible. Así, no se presenta tanto más una homogeneidad pensable de lo religioso, de lo que pudiéramos denominar una sistemática interna, que un despliegue lineal, en el que pudiéramos desplazar un cursor entre un menos y un más, un menos de emancipación o un más de tradicionalismo, por ejemplo. Más bien, enfrentamos surgimientos siempre nuevos que deben interpretarse. Eso es lo que ocurre hoy con las radicalidades religiosas, tales como el evangelismo o el islamismo, típicas de nuestro tiempo ${ }^{7}$ : son provocadas por la situación sociocultural presente, y su llegada al primer plano responde a dificultades sociales contemporáneas (especialmente, la desorientación y la globalización); y son productos de esta situación en su forma misma (pensemos, por ejemplo, en la unicidad de un significado, pese a que la tradición en que se inspiran haya teorizado lo contrario, como en el cristianismo; un fenómeno de racionalización interna que lleva a comprender y a presentar la posición religiosa como un saber ${ }^{8}$; la construcción de un pasado ideal, deshistorizado y desculturalizado; una socialización religiosa ajena a todo arraigo tradicional y a las mediaciones que allí se cristalizan; etc.).

Todo lo que acabo de esbozar debe conocerse. Y la Universidad debe ocuparse de ello. En efecto, deben conocerse estas diferencias, estas discontinuidades, estos surgimientos, con sus características propias en cada caso y lo que revelan. Conocer para alejarse de lo que surge a partir de reflejos espontáneos y de desconocimientos diversos, tanto en el pla-

7 Lo cual se escoge por estar directamente vinculado a lo aludido ; cf. Quel âge post-séculier? Religions, démocraties, sciences (Joan Stavo-Debauge, Philippe González et Roberto Frega dir.), Paris, EHESS, 2015 ; Samir Amghar, Le salafisme d'aujourd'hui. Mouvements sectaires en Occident, Paris, Michalon, 2011 ; las dos contribuciones de Maurice Borrmans sobre estos movimientos en islam, en Evangile, moralité et lois civiles / Gospel, Morality, and Civil Law (Joseph Famerée, Pierre Gisel et Hervé Legrand dir.), Berlin, Lit, 2015.

8 El creacionismo ofrece aquí una escena típica y es, además, una creación moderna, reactiva, ciertamente, pero moderna en todo lo que constituye tanto su tipo de posicionamiento en la sociedad como su propio contenido; ver, mencionado en este caso, también, en función del propósito de la presente contribución: Philippe GonzÁlez y Joan Stavo-Debauge, " Dominez la terre !”. Le créationnisme, du fondamentalisme à la désécularisation ", Archives de sciences sociales des religions $169,2015 / 1$, p. 351-576. 
no religioso como en el social. Conocer para comprender mejor y así poder acoger la alteridad que se entreteje en el corazón de todo lo humano -de lo cual lo religioso es un escenario particularmente apropiado-; una alteridad de la cual hacer algo, positivamente, y no para conjurar, ni erigirla en objeto de temor contra el cual defenderse. También -lo que no carece de importancia- conocer con el fin de estar mejor equipados para interpretar el presente, que vemos con demasiada frecuencia en función de pérdidas -que estimamos afortunadas o desafortunadas- o de beneficios vinculados a la novedad -de lo que podemos también regocijarnos o lamentarnos- y según oposiciones binarias que llevan a error, y que no permiten comprender lo que ocurre realmente.

\section{Lo religioso como síntoma}

En materias religiosas y para lograr un debate en la sociedad que sea provechoso por encontrarse articulado con lo que sucede realmente a través de la historia, así como en el corazón del presente, existen conocimientos que debemos registrar y que convendrá integrar a los saberes organizados. Esto no es menor en un tiempo de analfabetismo religioso, derivado de un período bastante prolongado de privatización en el que no todo es contenido sociopolítico, sino también sus consecuencias intelectuales, habiéndose relegado lo religioso a convicciones subjetivas, elecciones y juicios personales -cuando no reducido a los «bienes propios» de una institución separada, con sus discursos internos y sus autorreferencias- fuera de algún espacio de razón que permitiría dar cuenta de ello, y de discernir los desafíos presentes, así como de evaluarlos.

Pero en materias religiosas no solo se debe conocer o saber y, esto, en un horizonte amplio, que no es el de la conciencia espontánea de los actores ni de los creyentes. También, a raíz de ello, se debe interpretar lo que sucede y particularmente en relación a lo social, que constituye aquello con lo cual está en conflicto y que lo atraviesa. Lo religioso vale aquí como sintoma ${ }^{9}$, en sus propias formas y en lo que allí se encuentra cristalizado. Como lo habremos presentido en lo que señalé sobre lo

9 En esta materia, que he destacado con frecuencia, ver Véronique ALTGLAs, « La religion comme symptôme ", en Le religieux entre science et cité. Penser avec Pierre Gisel (Philippe GonZÁlez et Christophe Monnot dir.), Genève, Labor et Fides, 2012, p. 33-48. 
religioso difuso y las «espiritualidades laicas» o «sin Dios», con lo que en ellas se afirma prioritariamente de una búsqueda de equilibrio en la vida, que puede valer como un retorno de sabidurías antiguas e inspiradas en conocimientos arcaicos o esotéricos, convocando dimensiones no tomadas en cuenta en la oficialidad de los saberes autorizados, en este caso bajo sospecha de un giro tecnocrático y de una reducción del campo de los datos pertinentes.

En materia de síntoma, me referiré a dos fenómenos que coinciden en gran medida con el haz que despliega lo religioso en nuestras sociedades contemporáneas, a la vez que cristalizan allí la novedad, lo que ofrece momentos particularmente instructivos.

El primer fenómeno, ya mencionado, son las radicalidades religiosas como, por ejemplo, el evangelismo en el cristianismo, el islamismo o el salafismo en el islam. Dos tendencias -que pueden dar forma a movimientos organizados- que presentan paralelos sorprendentes. No en sus afirmaciones de primera instancia, ciertamente, sino que en el modo de posicionarse frente a la sociedad, reconstruir su pasado, desacreditar una historia efectiva para oponerle un modelo de los orígenes, devaluar las mediaciones (en el islam, el Derecho que es el fiq, en favor de una sharia religiosa que existiría directamente como código; en el evangelismo, todo lo que forma parte del orden que cristaliza la Iglesia, salvo lo comunitario ideal en negación de la institucionalización y, por ello, en favor de un momento bíblico autosuficiente). Aquí la visión es de entrada globalizada, y, por ello, uniformizada; con frecuencia, se recurre a Internet, no solamente como fuente directa de información, sino también como un lugar o un momento de formación. Como resultado, emerge de la «religión sin cultura», retomando la expresión acuñada por Olivier Roy ${ }^{10}$, donde no debe entenderse que la religión no estaría abierta a una cultura que la enfrenta y que tendría también sus propios valores - por reconocer-, sino en el sentido de que la religión no estaría intrínsecamente hecha de datos culturales, lo que sin embargo es y ha sido siempre así (en el cristianismo, incluso hemos teorizado sobre ello). Entonces, lo que se ha destacado actúa como contramodelo y se presenta como integral y en el mismo plano que aquello a lo que se opone y, por ello, como alternativa. Este punto, fundamental en mi opinión, refleja

10 La Sainte ignorance. Le temps de la religion sans culture, Paris, Seuil, 2008. 
un síntoma ${ }^{11}$. Y también en este caso existe la oportunidad de una verdadera novedad, aunque -como es casi siempre el caso- se niegue y nos digamos, deseemos y comprendamos como verdaderos fieles, a diferencia de los liberales adaptativos denunciados entonces.

El segundo fenómeno se refiere a lo religioso compensatorio. Se integra deliberadamente en la primera de las funciones que la religión puede asumir hoy en día, según Slavoj Zizek (aunque él prefiere la segunda): «En el nuevo orden mundial, dos roles son posibles para la religión: un rol terapéutico o un rol crítico. La religión o bien ayuda a las personas a integrarse cada vez más al orden existente, o procura afirmarse como una instancia crítica y señala lo que no funciona en este orden mundial como tal» ${ }^{12}$; en esta primera función, lo religioso adopta entonces formas típicas de lo contemporáneo, centradas ante todo en la persona y su «desarrollo personal»o apuntando a la progresión interior, excluyendo lo social que es dejado de lado y con lo que uno se acomoda o donde se ocupan diversos registros y espacios, por lo demás. Observemos que los proyectos de coherencia de hace algunos decenios, exigentes, incluso heroicos, no solo han abierto espacio a trayectorias múltiples en el corazón de lo social más desintegrado -o reducido a dinámicas técnicas de mero crecimiento cuantitativo hacia siempre más y siempre lo mismo, pese a la novedad proclamada constantemente, como un objeto ingenioso destinado a la rápida obsolescencia-, sino también a una multiplicidad situada en el centro mismo de las vidas individuales que se despliegan hoy al antojo de mundos, espacios y tiempos diferentes y más bien yuxtapuestos. Esto tiene su virtud y permite la singularidad en función de los itinerarios propios, pero deja lo social envuelto en insatisfacciones que se «vengan», lo social no pensado según una finalidad cualquiera, incluso que afecte a lo humano que se enmarca en ello, lo social neu-

11 Hoy, Christoph Theobald presenta una postura muy diferente -la menciono aquí solo como ejemplo- cuando habla, para circunscribir el Evangelio, con un "gesto»: un gesto se inscribe en efecto sobre un terreno dado, para ilustrar otra cosa o para provocar un desplazamiento, no para sustituir otro mundo (ello afecta lo que en ello hay de religioso, intrinsecamente, y lo que en ello hay de su relación con lo social), ver Le christianisme comme style, 2 vol., Paris, Cerf, 2007. Recordemos que esta era la perspectiva de Michel de Certeau, específicamente y con el término "gesto".Ver, entre muchos otros textos, La faiblesse de croire (Luce GIARD éd.), Paris, Seuil, 1987, p. 13-24.

12 Ver Slavoj ZizeK, La marionnette et le nain. Le christianisme entre perversion et subversion (2003), Paris, Seuil, 2006, p. 8. 
tralizado y, de hecho, homogeneizado, no exento de reducciones muy intensas, las más de las veces no conscientes, sin contar el daño hecho a la biósfera por causa de la dinámica ciega que allí opera ${ }^{13}$.

Visto en relación a lo social $-\mathrm{y}$ como se indica aquí sin un juicio moral-, lo religioso se cristaliza en dicho caso en una suerte de compensación $^{14}$. Girará en torno al ego ${ }^{15}$ (es el tiempo de los narcisismos), sin estar en disputa con lo social ni operar en este campo. Michel de Certeau ya había identificado este fenómeno al referirse a una religión en vías de "folclorización», sin una confrontación decisiva y creadora con lo real del mundo, lo que manifiesta como obstáculos y resistencia, un mundo que se propone y reinvindica la subversión interna.

Vemos que lo religioso, en las formas mismas que adopta -que conviene poner en perspectiva e interpretar-, puede ser objeto de un debate sobre lo social mismo, al que ilumina de manera indirecta y del que deja entrever síntomas.

4. El retorno de cuestiones de fondo, marginalizadas o reprimidas

Así, es preciso conocer, saber e interpretar lo que sucede. Pero, asimismo, es necesario reflexionar. Para ello, nos apoyaremos, en un primer momento -posteriormente será necesario actuar de manera deliberadamente más reflexiva-, sobre el eje de las represiones que existen escondidas en el corazón mismo del presente, que reflejan «retornos» algo explosivos $\mathrm{y}$, digamos, incomprensibles - las radicalidades religiosas son representación de ello- o que manifiestan cristalizaciones en forma de compensación, diversas acciones de supervivencia o, simplemente de vida. En otras palabras, releeremos los fenómenos que llegan al primer plano

13 Hay en este caso una fuente nueva de crítica a la modernidad, ver "Pensée écologique», en Dictionnaire de la pensée écologique (Dominique Bourg y Alain PAPAUX dir.), Paris, PUF, 2015, p. 756-759.

14 Como contexto al decir plausibilidad y oportunidad en un tiempo de «salida» de la religión o de lo teológico-político, ver Marcel GAUCHET, La religion dans la démocratie. Parcours de la lä̈cité, Paris, Gallimard, 1998, p. 89-110.

15 Ver el archivo de Jörg Stolz, Judith Könemann, Mallory Schneuwly Purdie, Thomas Englberger y Michaël KrÜGgeler, Religion et spiritualité à lt alii, RRalii, $d u$ séculier, Genève, Labor et Fides, 2015 a démocratie ?ngent, sans compter atteintes à la biosphères toujours m'ère de l'ego. Profils de l'institutionnel, de l'alternatif, du distancié et du séculier, Genève, Labor y Fides, 2015. 
como indicios de problemas, de dimensiones humanas y sociales (no se trata de un derecho de las convicciones y religiones, sino de realidades por reconocer y comprender, realidades que pueden ser especialmente impulsadas por lo religioso e incluso encontrar allí afinidad, pero sin ser reductibles, ni identificar datos propios que constituirían un ámbito exclusivo). Ello es causa de la pulsión, que puede traducirse en expresiones y construcciones diversas -tomadas del funcionamiento normal de instituciones, de discursos y de imaginarios-, que pueden así, ser marginados, o incluso, justamente, reprimidas y encontrarse actuando de manera diferida.

Partir del presente, de lo que en él está distorsionado y respecto de lo cual existen manifestaciones en términos de «retornos de represión» (o de lo cual se encuentra la evidencia de insatisfacciones de fondo) y discernir problemas, asumidos o no (y de maneras diversas, cuando es el caso, pues se trata aquí de un espacio fundamentalmente abierto) y que refieren a una disposición antropológica (incluidas las fuerzas de tipo pulsional), apela y se abre a un tratamiento genealógico.

Partiremos de «lo que nos ocurre» socioculturalmente, lo cual determina negativas, afirmaciones y búsquedas vinculadas a diversos afectos y a una cierta comprensión de lo que ocurre. A partir de ello, es posible una descripción en relación con desafíos expresables y que pueden desplegarse mediante una racionalidad pública.

La genealogía abre una perspectiva histórica, pero sin remitir a un desarrollo lineal, que podría parecer más seguro y menos abierto a disputa, pero que de hecho no es neutral. ¿Qué habría efectivamente de desarrollo lineal en las materias que nos ocupan? ¿Una racionalización creciente, como si la racionalidad fuera exclusivamente la de los saberes modernos y funcionamientos técnicos? ¿Una emancipación respecto de un modelo arcaico por superar, y en favor de una novedad que sería unilateralmente buena, sin ambivalencias fundamentales?

Si una puesta en perspectiva genealógica significa tomar en cuenta una historia, ella se construye y se despliega según un orden de problemas diagnosticados a partir de las dificultades o aporías del presente y frente a las cuales dispondremos de caminos diversos y sucesivos, objetos de puestas en escena que podrían considerarse equivocadas o correctas según las visiones históricas propuestas entonces, pero que, por una parte, son siempre sintomáticas $y$, por otra, dependen de lo operativo, efectivo y 
remiten a ello. Los problemas presentados, así como la historia de sus diversas disposiciones, serán formalizados e interpretados en este plano.

Agrego que, tal como se ha propuesto, una puesta en perspectiva genealógica supone una mirada que considere el largo plazo, que solo puede mostrar disposiciones de fondo diferentes, que pasan por discontinuidades reales, distintas de simples modificaciones con valorizaciones y negativas que toman forma sobre un terreno que es, a fin de cuentas, siempre el mismo, no descentrado e insuficientemente problematizado. La mirada desarrollada requerirá, además, emplear un contexto de amplio alcance, que muestre, más sincrónicamente esta vez, diferencias de civilizaciones o de sistemas simbólicos y que pueda desembocar en la comparación. A partir de una relectura de lo religioso como lugar de problemas inscritos en lo humano y lo social como tales -fuera de la conciencia de los actores, dentro de las religiones como de la sociedad de otros lugares-, se podrá entonces mostrar desafíos que aborden lo que constituye lo humano en su existencia singular e inscrita en el mundo, desafíos sobre los que es necesario reflexionar.

Estos desafíos han sido abordados por las religiones de manera diversa e incluso de un modo indirecto o, al menos, sin presentar motivos que estarían en pie de igualdad con lo que se afirma hoy en el corazón mismo del presente ni con lo que se puede descifrar como represiones. Reflexionar implica entonces presentar cuestiones transversales. A modo de ejemplo: el tipo de relación con el tiempo o el espacio; la manera de dar cuenta de la alteridad y de lo que existe en exceso, externo a lo religioso considerado o gestionado en su propia esencia; los modos de legitimación aplicados, el tipo de relación con el fundamento; la puesta en práctica o no de mediaciones, con sus regulaciones y procedimientos correspondientes. Ya señalé que las discontinuidades de disposiciones socioculturales que marcan toda historia y toda tradición muestran que lo religioso no es asunto de objetos fijos y aislables, sino que de puestas en escena, lo que remite a problemas, asumidos o diferidos. Las escenas propuestas, especialmente de un modo religioso, serán en este caso consideradas como maneras, en cada caso particular, de responder a cuestiones humanas de fondo y de todos. Así, sobre la cuestión de la trascendencia, que no es tanto lo que correspondería o satisfaría (el Dios de Abraham, de Isaac y de Jacob o el Dios de Descartes; Yavé o Alá; un Más Allá del ser o un principio onto-teológico o incluso una huella dejada sobre una asimetría de fondo; etc.), sino una manera, entre otras, de responder 
a aquello que excede o no es reductible a lo humano. Lo que puede hacerse de otro modo precisamente, por ejemplo, mediante «negociaciones» de tipo ritual provenientes de técnicas del cuerpo y el espíritu, sin reconocer algo trascendente con lo cual entraríamos en una relación que puede llegar hasta lo dramático del alma, tal como lo ha ilustrado especialmente el cristianismo. Y lo que puede ser también la ocasión para una negación basada en todo aquello que existe en exceso, en favor de un universo domesticable, más o menos dominable técnicamente, y de una sociedad secretamente o insidiosamente homogeneizada.

Decir trascendencia es entonces una manera particular de dar razón de una realidad más vasta, abierta y cargada de una problemática que la diversidad de maneras de dar forma a la vida en el mundo puede ayudar a perfilar. Podremos, entonces, experimentar con la tipologización presentando, para cada forma considerada, las respectivas fortalezas y debilidades en cuanto a lo que es, en cada caso, un hecho de lo humano.

El motivo de la trascendencia no era aquí más que un ejemplo. No está fuera del tiempo, pues para la perspectiva genealógica abierta, en verdad, conviene partir de los problemas del presente, apoyándose sobre el eje de represiones o cristalizaciones en forma de compensación. Ahora bien, en este contexto, lo que ocurre en torno al motivo de la trascendencia resulta significativo. En efecto, se establece un rechazo pues la trascendencia es vista y por ello denunciada, como heteronomía ${ }^{16}$, pero sin un desfase y según una inversión que dice más sobre los afectos de la época, que sobre los desafíos de fondo. Sobre todo, corremos el riesgo de caer en la «repetición», diferida, ciertamente, pero siempre igual, invertida o «reactiva». En nada se renueva, y siendo caricaturesco acerca del pasado de donde provenimos y superficial en cuanto al presente, y nos deja condenados a permanecer infructuosos y a invocar objetivos ideacionales e idealizados - «el hombre genérico» de la izquierda hegeliana o un republicanismo recurrente de la sociedad francesa-, en un contexto igualitario e igualizado ${ }^{17}$, o, por el contrario, a ser presas de la reafirmación de heteronomías que justamente reflejan la insatisfacción.

16 En lenguaje católico y especialmente en los debates que discurren en torno a la crisis modernista, se ha hablado de "extrinsecismo», objetado teológicamente, así como de «fideísmo» y "tradicionalismo».

17 En el caso de los elementos de la problemática de fondo referida en la perspectiva que presento, ver Pierre Manent, Situation de la France, Paris, DDB, 2015. 
Esto se encuentra unido al motivo de la trascendencia, como lo habremos presentido, nada menos que la cuestión del sujeto, sus formas y, hoy en día, su destino y, por ello, la visión de lo humano y su relación con lo social ${ }^{18}$. Una temática igualmente investida de reflejos espontáneos, donde se percibe una descalificación de todo antecedente y toda pertenencia en nombre de una autonomía validada sobre un trasfondo de historia demasiado corta; así, los rechazos que se articulan a este respecto bien pueden aparecer como legítimos, pero solo en el plano de lo que está en juego sin encontrarse, tampoco en este caso, ni descentrados, ni problematizados. Además, queda estancada toda cuestión de identidad $^{19}$, habiendo sido claramente neutralizada la cuestión de la simbolización, la relación con el tiempo, con el espacio, con el otro, consigo mismo, así como con las temáticas de la memoria, la tradición, los relatos actuales o futuros.

$\mathrm{Al}$ volver a reflexionar sobre ello, la unión entre el motivo de la trascendencia y la cuestión del estatus y la forma del sujeto ${ }^{20}$, revela en el fondo una imposición contemporánea hacia la homogeneización, progresiva pero profunda. Reforzada por una divulgación de imperativos técnicos y por una pregnancia de principios llamados éticos, pero que se refieren primeramente a las maneras de hacer, dejando de lado los problemas de fondo y toda alternativa fuerte ${ }^{21}$. No se recurre a lo heterogéneo aquí, lo que permitiría problematizar y transformar lo que hace casi naturalmente la organización de la sociedad y determina su curso; y queda poco espacio para la disidencia o la objeción de fondo. Agreguemos

18 Slajov ZizeK y Peter SLOTERdijk se unen, de manera distinta, a este ensamblaje, ver, respectivamente, Fragile absolu. Ou pourquoi l'héritage chrétien vaut-il d'être défendu, Paris, Flammarion, 2008, y La mobilisation infinie. Vers une critique de la cinétique politique [trad. de Eurotaoismus, 1989], Paris, Christian Bourgois ed., 2000.

19 De ahí la reacción de los pensadores antimodernos que combaten lo «políticamente correcto» y un "totalitarismo del pensamiento convencional», tales como Alain Finkielkraut o Eric Zemmour, aquí típicos: proponen ciertamente, a mi modo de ver, respuestas inadecuadas, pero ¡a buenas preguntas!

20 Además de las referencias ya dadas en el plano de los diagnósticos directamente sociales, remitiremos al trabajo de fondo llevado a cabo por Emmanuel LEvinas, en particular Autrement qu'être ou au-delà de l'essence (1974), Paris, Livre de poche, 2006.

21 Ver, en esta materia, el muy perspicaz Mark HunYADI, La tyrannie des modes de vie. Sur le paradoxe moral de notre temps, Lormond, Le Bord de l'eau, 2014. 
que la homogeneización incriminada se perfila en el horizonte de una crisis, incluso como el colapso de lo institucional, así como del orden de las mediaciones que la acompañaban. Allí se condensaba un tercero -entre la persona y el mundo-, necesario en la medida en que había exceso o que el sujeto no era puramente autoposición a partir de sí mismo. De ello, la escena religiosa lo testimonia incansablemente, tanto de manera indirecta como directa. Pero se deberá precisar lo que implica examinar las tradiciones en este marco conjunto, para ellas y para la teología que se articula en ellas, incluso cuando son vistas por ellas mismas.

En suma, lo contemporáneo tiende a no concederle derecho a la diferencia - no existe una afirmación verdadera de sí mismo, ni de una consistencia del mundo conformada de raíces por habitar y transformar-, salvo por la acomodación que, de hecho, desarma y remite al folclor lo que pertenecerá, desde entonces, a una tribu que la sociedad posmoderna puede integrar sin desviar, ni cuestionar, ni interrumpir su curso. El retorno de las cuestiones vinculadas a lo religioso o que hayan sido abordadas por las religiones, obstaculiza este despliegue y, siempre y cuando sea aprehendido y reflexionado según los juegos de diferenciación aquí esbozados, puede convertirse en una profunda renovación para lo humano y la sociedad.

5. Sobre un tratamiento diferenciado de lo social y una validación de las diferencias y de lo heterogéneo

El objetivo, la meta y el horizonte en este caso apuntan nada menos que a una renovación de lo social de todos. Trabajar las religiones y ver lo que ocurre en ellas ofrece la oportunidad de realizar un debate sobre la sociedad misma y no solamente sobre el lugar que se reserva o no a las religiones. Sin embargo, todo lo que he expresado hasta el momento lleva a negar que ello se haga conforme a un tratamiento único y bajo un solo ángulo, rápidamente dominado por lo universal cuya insuficiencia ha quedado claramente demostrada por las reacciones contemporáneas. El trabajo debe llevarse a cabo en articulación con cada una de las tradiciones en juego, que no deben ser ni eliminadas ni dejadas al margen. Pues ellas cristalizan el hecho de que los «fines» del hombre no pueden reducirse al conjunto de lo social como tal, sino que remiten a lo heterogéneo, a fortiori cuando son religiosos y son necesariamente plurales. 
Como habrá quedado claro, considero que la particularidad de las cristalizaciones religiosas - tal como las cristalizaciones sociales de otros ámbitos- es ineludible. Conviene partir de ella y volver a ella ${ }^{22}$. Constituye el único lugar donde lo humano responde realmente de sí, de lo que lo provoca y lo hace ser. Y no es por azar que sea asimismo el único modo sobre el cual se constituye lo religioso y lo social, salvo por los sueños y la ilusión. La particularidad es, en definitiva, ineludible debido a que contiene lo heterogéneo fundamental y a que en sus propias diferencias, incluso en el corazón del mundo, las cristalizaciones religiosas o más globalmente sociales, no pueden más que darse de manera heterogénea unas y otras.

No valido este hecho para abrirnos a particularismos autorreferidos y, por ello, yuxtapuestos unos a otros. Lo descrito, por el contrario, aboga porque cada uno sea expuesto a la alteridad y que se abra el debate a partir de las diferencias manifestadas, movilizando lo transversal y, así, lo común ${ }^{23}$, pero no bajo la forma de tal o cual elemento que pueda encontrarse en cada uno, ni tampoco mediante un rebasamiento en favor de una posición conciliadora, más alta o más profunda.

En favor de la existencia humana que se desarrolla verdaderamente y de una vida en sociedad que está siempre renovándose, en materias religiosas, será necesario entonces mantener una doble mirada ${ }^{24}$. Según una diferencia irreductible de ejes, interrogaciones y problemáticas, aunque no sin que cada uno de estos ejes de trabajo se enriquezca indirectamente con lo que aporta el otro eje.

22 Tocamos aquí un punto - una elección- decisivo para mí; más aún, consiste en un tema recurrente en mi "Leçon d'adieu» al momento de dejar mi carga académica: «Résistances des particularités et pièges de l'universel. Pour un usage subversif des corps, des traditions et des frontières», en Mises en scène de l'humain. Sciences des religions, philosophie, théologie (Jacques Ehrenfreund et Pierre Gisel dir.), Paris, Beauchesne, 2014, p. 227-247 (traducción italiana de la lección: "Dell'universale e delle particolarità ", Il Regno LVIII, No 1142, 2013, p. 249-256).

23 En materia de gestión social de lo «común», remitiría con gusto a Jean-Luc Nancy, ver Penser en commun? Un «rapport sans rapport». Jean-Luc Nancy y Sarah Kofman lecteurs de Blanchot (Isabelle Ullern et Pierre Gisel dir.), Paris, Beauchesne, 2015.

24 Precisé este punto en "Une double vocation de la théologie, interne et externe. Ordres différents et compatibilité», Études théologiques et religieuses 88, 2013/3, p. 375-390. 
Así se abordará cada tradición religiosa en sí misma, en lo que ofrece, en su historia, sus propias transformaciones, su manera de hacer frente a los desafíos de los tiempos (y puede ser responsabilidad del Estado favorecer este trabajo ${ }^{25}$. Pero se realizará haciendo resonar, en la esencia misma de sus realidades más propias -simbólicas, rituales, doctrinales y otras-, las cuestiones humanas a las que de hecho responde y a las cuales da una forma particular ${ }^{26}$, no simplemente repitiendo el dato, ni dejándolo a lo que sería el espacio propio. En el cristianismo, una de las tareas de la Teología será ocuparse de ello.

Al mismo tiempo, se trabajará fuera de todo espacio de coordenadas similares (ni en cuanto a los objetos, ni en cuanto las problemáticas), se abordará la escena religiosa global en función de la sociedad misma y del descentramiento que ella representa en relación con lo religioso y sus diversas cristalizaciones. Esto responde al afán de describir la diversidad, sus mutaciones y lo que la trabaja, lo que implicará tomar en cuenta también la cuestión de cómo agenciarlo mejor para lo humano, pero dada la perspectiva de conjunto empleada, con miras a regular, no a reprimir, las diferencias e incluso a favorecer su expresión, según «fines» que rebasen lo social y en que presidan las diversas instancias que atraviesan la realización de lo humano en la esencia del mundo. Aquí, las diferencias que se manifiestan han tomado y toman rostros también religiosos. Tendremos en cuenta esto, positivamente, en lo que pueda «traducirse» en términos seculares (por ejemplo, desde un descanso sabático impuesto por una ley divina a una consideración sobre los beneficios para lo humano de ritmos temporales acondicionados). Pero lo que se resiste a una traducción o bien no ha sido traducido, y que cristaliza lo heterogéneo, será también descifrado positivamente, primero como un recordatorio sano -independientemente de sus formas, que pueden ser insanas o peligrosas-, un recordatorio de validar en cada caso con respecto a una sociedad que tiende a homogeneizarse en función del imperativo de asumir la responsabilidad; y dicha heterogeneidad estará, en segundo lugar, relacionada con motivos antropológicos y sociales de mayor alcance (un ejemplo puro de ello: la circuncisión judía, que no ha sido trasladada, ni tampoco «traducida», excepto por la metabolización

25 Es bueno para la sociedad global que las tradiciones tengan un lugar de trabajo en ellas, por ejemplo, en las facultades de teología cristiana, islámica u otras.

26 Para ahondar en la materia, ver $D u$ religieux, du théologique et du social. Traversées et déplacements, Paris, Cerf, 2012, en particular p. 106-107. 
extrema y a decir verdad dulcificadora, al tiempo que presenta motivos, tales como el engendramiento y la generación que la sociedad moderna justamente no considera). Observemos que, de esta tarea, determinada por las coordenadas de lo social de todos, la Teología en su forma cristiana puede adoptar también, como tarea asumida como propia, pero irreductiblemente diferente a la primera que ya señalé, esta segunda tarea que no puede ni debe comprenderse como una prolongación de la primera, incluso a título de una abertura para el bien de todos, lo que proviene de la buena voluntad, pero es de hecho diabólico.

6. Sobre un trabajo reflexivo acerca de las realidades religiosas y sociales

Lo que he intentado señalar demuestra que lo religioso, y lo que sucede a propósito de ello, presenta grandes desafíos para lo humano y lo social, que deben actualizarse y evaluarse. Para descifrarlos, no nos situaremos en un plano "proposicional», y ciertamente tampoco en cuanto a su relación directa con el objeto ${ }^{27}$. Cuando está presente -lo que no es siempre el caso-, lo "proposicional» pertenece intrínsecamente a la expresión religiosa $-\mathrm{y}$ así tal afirmación de fe o tal dogma-, pero convendrá precisar de qué orden depende y lo que asegura en cada caso, en principio legítimamente, pues las modalidades concretas están reservadas. Por mi parte, no descarto ninguna de las positividades de lo religioso (en favor de un espiritualismo modernizante que ha podido sostener una forma tal de protestantismo liberal) e incluso pienso que deben abordarse como tales y que no debe dejarse lo religioso ya sea a la pura convicción, ya sea a las consideraciones sociológicas, históricas y otras que se mantendrán en lo descriptivo puro. Sin embargo, es necesario precisamente que no se malentienda su estatus y su función, que son además diferentes.

Recordemos que no replegar sobre lo «proposicional» la cuestión de los desafíos de fondo aludidos -desafíos por discernir y que brindarán la oportunidad de realizar una evaluación- es tradicional en la historia del pensamiento o de la teología cristiana. Guarda relación con esto último que en materia de fe, por ejemplo, se precisara que la «verdad» no era función de un objeto que sería del mismo tipo que los objetos

27 La precisión vale aquí como demarcación con respecto a numerosos defensores de la filosofía analítica que intervienen hoy en el campo de las cuestiones religiosas. 
del mundo y con el cual nos relacionaríamos mediante el mismo modo. El conocimiento natural que tenemos de ello incluso debe aumentarse más, lo «sobrenatural»; tradicionalmente, aquello a lo que nos remite la fe constituye, en efecto, otro orden y demanda una modalidad de relación con el mundo -llamada «teologal»-, y el mundo es aquí justamente el único objeto en juego, conforme al significado habitual del término objeto.

Destaquemos que decir desafíos - a fortiori proceder a la evaluacióndepende de lo reflexivo y vale a título de un segundo trabajo, que supone la problematización, por medio de las positividades o las efectividades dadas. Es una tarea de tipo filosófico, pero que será ejercida en interacción con las ciencias humanas y sociales (en particular las «ciencias de las religiones»), que pueden ser problematizantes -e incluso deben serlo- $y$ cuyos conocimientos propuestos deben en cualquier caso ser considerados en el marco de un trabajo reflexivo. ¿Es esta una tarea distinta que aquella de la teología, que es igualmente reflexiva, a fortiori en el sentido de que se despliega a título de una «teología fundamental», en otro tiempo denominada «especulativa», y que se distingue de la "positiva» o de lo que Schleiermacher llamaba precisamente «teología filosófica», distinta de la «dogmática»? Tal vez no. Pero a fin de cuentas la cuestión en este caso no es tanto satisfacer las costumbres y los métodos disciplinarios, en especial pues se abre hoy un camino a una verdadera neutralización (no es casualidad que yo haya denominado transversales ${ }^{28}$ a estas cuestiones), sino que asegurar un orden de cuestionamiento; este orden será además transversal no solamente respecto del ejercicio de las disciplinas constituidas y reconocidas, sino que de las realidades humanas y sociales en cuestión. Lo mismo cabe afirmar cada vez que, por causa de lo religioso y, también, de manera patente, no nos remite a un más o a un junto a, reservado, sino que lo religioso se refiere a lo que está hecho, lo que se asume, que responde -y al mismo tiempo en forma de respuestaa usos que llevan a un paso más allá o de otra forma a un dato.

28 Aquí se requerirá la «indisciplina», en el doble sentido del término. Ver mi ponencia presentada casi en paralelo, "La pertinencia teológica del pensamiento de Michel de Certeau. La indisciplina de la interdisciplinariedad», en el coloquio "Michel de Certeau: en el cruce de las ciencias del hombre. En torno a la historia del cristianismo moderno, sujeto e institución", en Santiago de Chile (Pontificia Universidad Católica de Chile y Universidad Alberto Hurtado), 3 nov. 2015. 
Articulado con el conjunto de los fenómenos que he indicado, el cuestionamiento al que se apunta permite abrir un espacio de debate sobre lo que está en cuestión, comprendiendo los desafíos de fondo; un debate en el marco de una racionalidad pública-creamos o no, seamos o no sensibles a lo religioso-, y un debate que, a fin de cuentas, aborde lo humano y lo social. Así expuesto, dicho debate podrá abrirse a una evaluación razonada, tanto en el plano social, como religioso. Una evaluación asumida singularmente, pero de la cual podamos rendir cuentas. Por consiguiente, nos pronunciamos acerca de ello. ¿Se trata pues de una cuestión de verdad sobre la cual decidimos y respondemos? Sí, si así lo deseamos, pero diferente de las expresiones directas -religiosas y otras- y, por lo tanto, cada cual deberá sujetarse a un régimen específico de razonamiento, articulado según lo que se despliega y apropiado al tipo de desafío de que se trate. 